\title{
CORRELACIÓN ENTRE EL DESARROLLO DE LAS INTELIGENCIAS \\ MÚLTIPLES Y EL RENDIMIENTO ACADÉMICO DE LOS ALUMNOS DEL \\ CENTRO DE ESTUDIOS PREUNIVERSITARIO (CEPU) 2007 - II DE LA UNIVERSIDAD NACIONAL JORGE BASADRE GROHMANN DE TACNA
}

\author{
Responsable: Mgr. Humberto Vargas Pichon \\ Miembros : MSC. Julio Fernandez Prado \\ Lic. Augusto Becerra Castañeda
}

\section{RESUMEN}

El presente trabajo de investigación es un estudio no experimental de tipo correlacional, de corte transversal, que se efectuó durante el periodo Abril 2007 a Abril 2008 en el Centro de Estudios Preuniversitarios (CEPU) 2007- II de la Universidad Nacional Jorge Basadre Grohmann de Tacna.

Mediante el objetivo general se pretendió "Establecer una correlación entre el Desarrollo de las Inteligencias Múltiples y el Rendimiento Académico de los alumnos del Centro de Estudios Preuniversitarios (CEPU) 2007-II de la Universidad Nacional Jorge Basadre Grohmann de Tacna", en respuesta a la interrogante ¿En qué medida existe una correlación entre el desarrollo de las inteligencias múltiples y el Rendimiento Académico de los alumnos del Centro de Estudios Preuniversitarios (CEPU) 2007 - II de la Universidad Nacional Jorge Basadre Grohmann de Tacna?, sosteniendo la hipótesis de que: "Existe una correlación entre el desarrollo de las Inteligencias Múltiples y el Rendimiento Académico de los alumnos del Centro de Estudios Preuniversitarios (CEPU) 2007-ll de la Universidad Nacional Jorge Basadre Grohmann de Tacna".

El diseño muestral fue probabilístico sistemático; y considerando el muestreo estratificado se trabajó sobre una población de 1818 alumnos y con una muestra de 279 alumnos del Centro de Estudios Preuniversitarios (CEPU) 2007- II de la Universidad Nacional Jorge Basadre Grohmann de Tacna. Para la determinación del desarrollo de las inteligencias múltiples se utilizó como instrumento un Inventario de Inteligencias Múltiples y para la determinación del Rendimiento Académico se utilizaron dos (02) exámenes del CEPU para los cuatro (04) canales de los alumnos conformantes de la muestra.

Asimismo, se utilizaron el Programa Estadistico SPSS V-11.5 y el Microsoft Excel XP 2003 para el procesamiento de datos y la elaboración de los cuadros y gráficos estadísticos, así como la prueba de hipótesis correspondiente, lo que nos permitió obtener la conclusión principal y conclusiones especificas correspondientes.

\section{ABSTRACT}

The present Work of Investigation is a nonExperimental study of corelational type, of cross section, that in center took place during the period April 2007 to April 2008 of Studies Preuniversitarios (CEPU) 2007 - II of the Nacional University Jorge Basadre Grohmann of Tacna.

By means of the general mission it was tried "To establish a correlation between the Development of Multiple Intelligences and the Academic Yield of the Students of the Training center Preuniversitarios (CEPU) 2007 - II of the Nacional University Jorge Basadre Grohmann of Tacna", in answer to the question To what extent exists a correlation between the development of multiple intelligences and the Academic Yield of the students of the Training center Preuniversitarios (CEPU) 2007 - II of the Nacional University Jorge Basadre Grohmann of Tacna. Maintaining the hypothesis that: "A correlation between the development Multiple Intelligences and the Academic Yield of the students of the Training center Exists Preuniversitarios (CEPU) 2007 - II of the Nacional University Jorge Basadre Grohmann of Tacna".

The design sample was probabilistic systematic and considering the sampling stratified Preuniversitarios (CEPU) 2007 - II of the Nacional University worked on a population of 1818 students and with a sample of 279 students of the Training center Jorge Basadre Grohmann of Tacna. For the determination of the development of multiple intelligences he was used as instrument an Inventory of Multiple Intelligences and for the determination of the Academic Yield he was used two (02) examinations of the CEPU for the four (04) channels of the conformantes students of the sample.

Also, he was used Statistical Program SPSS V-11.5 and Microsoft Excel XP 2003 for the data processing and statistical elaboration of the pictures and graphs as well as the test of corresponding hypothesis, which allowed us to obtain the main conclusion and corresponding specific conclusions. 


\section{INTRODUCCIÓN}

Probablemente una de las dimensiones más importantes en el proceso de enseñanza aprendizaje lo constituye el Rendimiento Académico del alumno. Cuando se trata de evaluar el rendimiento académico y cómo mejorarlo, se analizan en mayor o menor grado los factores que pueden influir en él, generalmente se consideran, entre otros, factores socioeconómicos, la amplitud de los programas de estudio, las metodologías de enseñanza utilizadas, la dificultad de emplear una enseñanza personalizada, los conceptos previos que tienen los alumnos, así como el nivel de pensamiento formal de los mismos.

Sin embargo, el presente trabajo trata de demostrar que existe una correlacion entre el Desarrollo de las Inteligencias Múltiples (IM) y el Rendimiento Académico (RA) de los alumnos del Centro de Estudios Preuniversitarios (CEPU) 2007-II de la Universidad Nacional Jorge Basadre Grohmann de Tacna.

\section{DESARROLLO DELTEMA}

\section{Cuadro de la Teoría de las Inteligencias Múltiples.}

\begin{tabular}{|l|l|}
\hline Inteligencia & Componentes centrales \\
\hline Lingüistica & $\begin{array}{l}\text { Sensibilidad a los sonidos, la estructura, los significados y las } \\
\text { funciones de las palabras y el lenguaje }\end{array}$ \\
\hline $\begin{array}{l}\text { Lógica- } \\
\text { matematica }\end{array}$ & $\begin{array}{l}\text { Sensibilidad y capacidad para discernir los esquemas } \\
\text { numéricos o logicos; la habilidad para manejar cadenas de } \\
\text { razonamientos largas. }\end{array}$ \\
\hline Espacial & $\begin{array}{l}\text { Capacidad para percibir con precisión el mundo visual y } \\
\text { espacial, y la habilidad para efectuar transformaciones en las } \\
\text { percepciones iniciales que se hayan tenido. }\end{array}$ \\
\hline $\begin{array}{l}\text { Corporal- } \\
\text { Kinetica }\end{array}$ & $\begin{array}{l}\text { Habilidad para controlar los movimientos del cuerpo y manejar } \\
\text { objetos con destreza. }\end{array}$ \\
\hline Interpersonal & $\begin{array}{l}\text { Capacidad para discernir y responder de manera adecuada a } \\
\text { los estados de ánimo, los temperamentos, las motivaciones y } \\
\text { los deseos de otras personas. }\end{array}$ \\
\hline Intrapersonal & $\begin{array}{l}\text { Acceso a los sentimientos propios y habilidad para discernir las } \\
\text { emociones intimas, conocimiento de las fortalezas y } \\
\text { debilidades propias. }\end{array}$ \\
\hline Musical & $\begin{array}{l}\text { Habilidad para producir y apreciar ritmo, tono y timbre; } \\
\text { apreciación de las formas de expresión musical. }\end{array}$ \\
\hline Naturalista & $\begin{array}{l}\text { Habilidades de ordenación y clasificación, reconocimiento de } \\
\text { secuencias de desarrollo, asi como la formulación de hipótesis, } \\
\text { aplicados en forma práctica en el conocimiento del medio. }\end{array}$ \\
\hline
\end{tabular}

1. Procesamiento y Análisis de los de datos

\section{- Análisis de coeficiente de correlación de Pearson respecto a las hipótesis específicas}

Primera hipótesis especifica:Este análisis permitirá probar si existe una correlación entre el desarrollo de las Inteligencias Corporal Cinética, Naturalista e Interpersonal (IM) y el Rendimiento Académico (RA) en el Canal 01 de los alumnos del Centro de Estudios Preuniversitarios (CEPU) 2007- II de la
Universidad Nacional Jorge Basadre Grohmann de Tacna.

En nuestro caso, el software estadístico SPSS nos presenta los siguientes resultados:

Correlaciones:

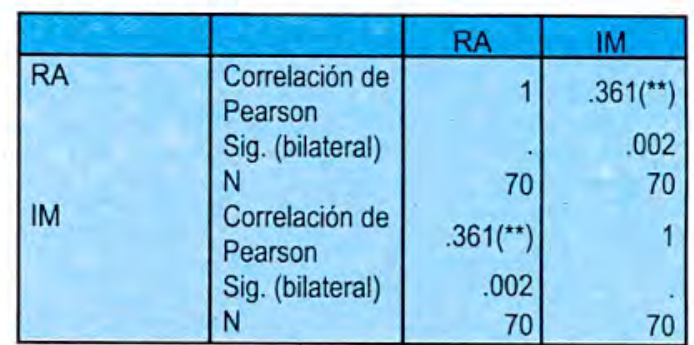

* La correlación es significativa al nivel 0,01 (bilateral).

\section{Por lo tanto: se acepta la hipótesis} específica $\mathrm{H} 1$.

Segunda hipótesis específica:Este análisis permitirá probar si existe una correlación entre el desarrollo de las Inteligencias Lógico Matemática, Intrapersonal, Corporal Cinética y Espacialvisual (IM) y el Rendimiento Académico (RA) en el Canal 02 de los alumnos del Centro de Estudios Preuniversitarios (CEPU) 2007-II de la Universidad Nacional Jorge Basadre Grohmann de Tacna.

En nuestro caso, el software estadístico SPSS nos presenta los siguientes resultados:

Correlaciones:

\begin{tabular}{|l|l|r|r|}
\hline & & \multicolumn{1}{|c|}{ RA } & \multicolumn{1}{|c|}{ IM } \\
\hline RA & Correlación de & 1 & $.468\left(^{* *}\right)$ \\
& Pearson & & .000 \\
& Sig. (bilateral) & & 65 \\
IM & N & 65 \\
& Correlación de & $.464\left({ }^{(*)}\right)$ & 1 \\
& Pearson & .000 & \\
& Sig. (bilateral) & 65 & 65 \\
\hline
\end{tabular}

** La correlación es significativa al nivel 0,01 (bilateral).

\section{Por lo tanto: se acepta la hipótesis especifica $\mathrm{H} 2$.}

Tercera hipótesis especifica:

Este análisis permitirá probar si existe una correlación entre el desarrollo de las Inteligencias Lingüistica e Interpersonal (IM) y el Rendimiento Académico (RA) en el Canal 03 de los alumnos del Centro de Estudios Preuniversitarios (CEPU) 2007-II de la Universidad Nacional Jorge Basadre Grohmann de Tacna.

Nuestro análisis ha utilizado el nivel de significación del 0,05.

En nuestro caso, el software estadístico 
SPSS nos presenta los siguientes resultados:

Correlaciones:

\begin{tabular}{|l|l|r|r|}
\hline & & RA & \multicolumn{1}{|c|}{ IM } \\
\hline RA & Correlación de & 1 & $.400(* *)$ \\
& Pearson & & .000 \\
& Sig. (bilateral) &. & 90 \\
IM & Correlación de & 90 & $.400\left(^{* *}\right)$ \\
& Pearson & 1 \\
& Sig. (bilateral) & .000 & \\
& N & 90 & 90 \\
\hline
\end{tabular}

** La correlación es significativa al nivel 0,01 (bilateral).

Por lo tanto: se acepta la hipótesis específica $\mathrm{H} 3$.

Cuarta hipótesis especifica:Este análisis permitirá probar si existe una correlación entre el desarrollo de las Inteligencias Lógico Matemática e Interpersonal (IM) y el Rendimiento Académico (RA) en el Canal 04 de los alumnos del Centro de Estudios Preuniversitarios (CEPU) 2007-II de la Universidad Nacional Jorge Basadre Grohmann de Tacna.

Nuestro análisis ha utilizado el nivel de significación del 0,05.En nuestro caso, el software estadístico SPSS nos presenta los siguientes resultados:

\begin{tabular}{|l|l|r|r|}
\hline & & \multicolumn{1}{|c|}{ RA } & \multicolumn{1}{c|}{ IM } \\
\hline RA & Correlación de & 1 & .225 \\
& Pearson & & .101 \\
& Sig. (bilateral) & 54 & 54 \\
N & Correlación de & .225 & 1 \\
& Pearson & .101 & \\
& Sig. (bilateral) & 54 & 54 \\
\hline
\end{tabular}

La correlación NO es significativa.

Por lo tanto: se rechaza la hipótesis especifica $\mathrm{H} 4$ y se acepta la hipótesis nula Ho correspondiente.

Análisis de coeficiente de correlación de Pearson respecto a la hipótesis general:Este análisis permitirá probar si existe una correlación entre el desarrollo de las Inteligencias Múltiples (IM) y el Rendimiento Académico (RA) de los alumnos del Centro de Estudios Preuniversitarios (CEPU) 2007-II de la Universidad Nacional Jorge Basadre Grohmann de Tacna. En nuestro caso, el software estadístico SPSS nos presenta los siguientes resultados:

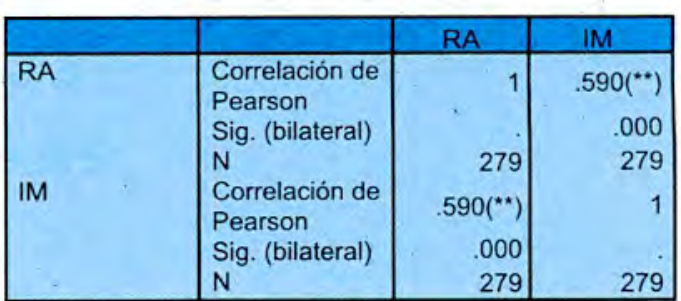

** La correlación es significativa al nivel 0,01 (bilateral). Por lo tanto: se acepta la hipótesis general $\mathrm{Hi}$.

\section{CONCLUSIONES}

1.El análisis de correlación de Pearson nos permite aceptar la hipótesis general $\mathbf{H}_{\mathbf{i}}$ de la investigación, esto es, "Existe una correlación entre el desarrollo de las Inteligencias Múltiples y el Rendimiento Académico de los alumnos del Centro de Estudios Preuniversitarios (CEPU) 2007-II de la Universidad Nacional Jorge Basadre Grohmann de Tacna".

2.El análisis de correlación de Pearson nos permite aceptar la hipótesis específica $\mathrm{H}_{1}$ de la investigación, esto es, "Existe una correlación entre el desarrollo de las Inteligencias Corporal Cinética, Naturalista e Interpersonal y el Rendimiento Académico en el Canal 01 de los alumnos del Centro de Estudios Preuniversitarios (CEPU) 2007-II de la Universidad Nacional Jorge Basadre Grohmann de Tacna".

3.El análisis de correlación de Pearson nos permite aceptar la hipótesis especifica $\mathbf{H}_{2}$ de la investigación, esto es, "Existe una correlación entre el desarrollo de las Inteligencias Lógico Matemática, Intrapersonal, Corporal Cinética y Espacial-visual y el Rendimiento Académico en el Canal 02 de los alumnos del Centro de Estudios Preuniversitarios (CEPU) 2007-II de la Universidad Nacional Jorge Basadre Grohmann de Tacna".

4.El análisis de correlación de Pearson nos permite aceptar la hipótesis especifica $\mathrm{H}_{3}$ de la investigación, esto es, "Existe una correlación entre el desarrollo de las Inteligencias Lingüistica e Interpersonal y el Rendimiento Académico en el Canal 03 de los alumnos del Centro de Estudios Preuniversitarios (CEPU) 2007-II de la Universidad Nacional Jorge Basadre Grohmann de Tacna".

5. El análisis de correlación de Pearson nos permite $\mathbf{N O}$ aceptar la hipótesis especifica $\mathbf{H}_{4}$ de la investigación, por consiguiente se acepta la hipótesis alternativa $\mathbf{H}_{0}$, esto es, "No existe una correlación entre el desarrollo de las Inteligencias Lógico Matemática e Interpersonal y el Rendimiento Académico en el Canal 04 de los alumnos del Centro de Estudios Preuniversitarios (CEPU) 2007-II de la Universidad Nacional Jorge Basadre Grohmann de Tacna". 


\section{RECOMENDACIONES}

Que investigaciones futuras relacionadas con la presente investigación puedan ser ampliadas y profundizadas y de Tipo Experimental, de tal manera que se obtengan resultados y propuestas orientadas a mejorar las condiciones de desarrollo personal de los estudiantes del Centro de Estudios Preuniversitarios de la Universidad Nacional Jorge Basadre de Tacna.

Creación de un Departamento de Psicopedagogia en donde se planifique e implementen evaluaciones psicológicas a sus alumnos desde su ingreso con el fin de conocer sus habilidades intelectuales y emocionales, así como sus deficiencias, de tal manera que puedan plantearse los correctivos necesarios.

EI CEPU de la UNJBG debe desarrollar programas y talleres de autoestima y motivación profesional que puedan significar un aporte para el desarrollo personal y motivación profesional de sus estudiantes. Las asignaturas del CEPU de la UNJBG deben propiciar actividades que contribuyan al desarrollo de habilidades intelectuales y emocionales de los estudiantes.

Que los docentes se informen e indaguen sobre sus propias inteligencias múltiples y las de sus alumnos, esto posibilitará una mejor selección de estrategias metodológicas.

Considerando que las inteligencias múltiples nos son definitivas o estables para cada individuo, el docente debe hacer uso de diversas estrategias metodológicas de enseñanza.

Informar a los alumnos sobre sus Inteligencias Múltiples más predominantes en ellos, asi como de estrategias para mejorarlas, a fin de obtener mejores resultados en su aprendizaje.

Explicar a los padres de familia sobre las Inteligencias Múltiples más predominantes en sus hijos, afín de que les brinden el apoyo adecuado y obtener asi mejores resultados con ellos.

\section{BIBLIOGRAFIA}

Andreani, D. (1975). Aptitud Mental y Rendimiento Escolar. (1 ${ }^{\text {a }}$ Edic.). Barcelona: Herder.
Armstrong, T. (2 001). Inteligencias Múltiples: Cómo descubrirlas y estimularlas en sus hijos. ( $1^{a}$ Edic. en castellano). Bogotá: Norma.

Carreño, F. (1 998). Instrumentos de medición del rendimiento escolar. (2 ${ }^{\mathrm{a}}$ Edic.). México: Trillas.

Garcia, J. (1 994). Bases Pedagógicas de la Educación: Guia práctica para educadores. $\left(1^{\mathrm{a}}\right.$ Edic.). Madrid: Síntesis.

Hardner, H. (1 993). Inteligencias Múltiples: La Teoria en la Práctica. ( $1^{\mathrm{a}}$ Edic. En Castellano). Barcelona: Paidós.

Hardner, H. (1987). Estucturas de la Mente: $\left(1^{\mathrm{a}}\right.$ Edic. en castellano). México: Fondo de Cultura Económica.

Navarro E. y Soto A. (2004). ¿Cómo estimular las inteligencias múltiples en el proceso de enseñanza y aprendizaje? Lima:Abedul.

Shneider, S. (2004). Las Inteligencias Múltiples y el Desarrollo Personal. Colombia: Printer Colombiana S.A.

Lapalma, F. (s/f). La Teoria de las Inteligencias Múltiples y la Educación.

Recuperado de,

http://www.turemanso.com.ar/fuego/educacio n/multiples2.html

[2007: 03 de Enero].

Aceros, J., Angarita S. y Campos O.

Depresión y Rendimiento académico

Recuperado de,

http://www.psicopedagogia.com/articulos/?arti culo $=311$

[2007: 08 de Enero].

Comisión de Ciencia y Tecnologia (1 998). Dictamen de los proyectos de ley $N^{\circ} 1759 / 96$ CRy 3340/97-CR.

Recuperado de,

http://www.congreso.gob.pe/comisiones/1997/ ciencia/17593340.htm

[2007: 02 de Diciembre]

Dirección Nacional de Educación Básica Regular-EBR (s/f). Diseño Curricular Nacional de Educación Básica Regular

Recuperado de,

http://destp.minedu.gob.pe/secundaria/nwdes/ discurna1.htm 